\title{
Editorial
}

\section{Circulating biomarkers in cardiovascular disease}

\author{
Erik Ingelsson* \\ Department of Medical Epidemiology and Biostatistics, Karolinska Institutet, Stockholm, Sweden
}

According to the Merriam-Webster Online Dictionary, a biomarker is 'a distinctive biological or biologically derived indicator (as a metabolite) of a process, event, or condition (as aging, disease, or oil formation)' [1]. A biomarker can be structural, such as mammography or a coronary calcium scan, or functional, such as glomerular filtration rate or blood pressure, but the term biomarker is most commonly used for describing measureable serological or circulating biological entities, such as proteins or metabolites. The purposes of measuring a biomarker vary, but include help in identification of individuals at increased risk for disease, screening for subclinical disease in asymptomatic individuals, diagnosis of existing disease in symptomatic patients, monitoring of treatment results in patients on therapy, or prognostication of recurrence or sequelae of disease.

The purpose of this special issue of 'Disease Markers' is to give a comprehensive overview of the current state of knowledge regarding circulating biomarkers in cardiovascular disease. The main focus of the eight articles is the use of biomarkers in risk stratification of healthy individuals or early detection of subclinical disease, but some of the contributions also discuss other uses of biomarkers, such as prognostication in individuals with prevalent disease. In cardiovascular medicine, there is a long history of using biomarkers

*Corresponding author: Erik Ingelsson, MD, PhD, Department of Medical Epidemiology and Biostatistics, Karolinska Institutet, Box 281, SE-171 77 Stockholm, Sweden. Tel.: +46 8 52482334; Fax: +46 8 314975; E-mail: erik.ingelsson@ki.se. for risk stratification. The blood lipid profile, including cholesterol fractions, was one of the first biomarkers to be established as a risk factor for cardiovascular disease [2]. Cholesterol fractions along with a set of other established cardiovascular disease predictors (i.e. age, sex, blood pressure, smoking and diabetes) have been included in multivariable risk stratification instruments, or scoring systems, such as the Framingham Risk Score [3] and SCORE [4]. For a novel biomarker to prove clinically useful in risk prediction, it should be demonstrated that it adds risk information over and beyond these readily available established risk predictors. It is important to emphasize that a high relative risk of CVD associated with a new biomarker after adjusting for established risk factors does not necessarily translate into better risk prediction. To address this, we need to use other statistical methods, for example discrimination metrics (such as C-statistics), but also measures of calibration (addressing how close the predicted risks are to the actual observed risks) and reclassification (addressing whether the model including the novel biomarker changes a person's risk sufficiently to move him or her to a different risk category, which could alter management decisions).

In the first article in the issue, Wood and Greenland discuss statistical approaches that can be used to evaluate additive predictive value of novel biomarkers and exemplify these methods in with clinical research examples in an intuitive manner. van Wijk, Stroes and Kastelein give a brief overview of lipid metabolism to go on discussing the epidemiological data for total, LDL- and HDL-cholesterol along with the role of 
triglycerides and the apolipoprotein B-A-I ratio in cardiovascular disease. Sarwar, Thompson and Di Angelantonio discuss the role of inflammatory biomarkers in cardiovascular disease, and provide many important methodological insights for interpretation of current data on such markers. Hildebrandt summarizes the current knowledge regarding natriuretic peptides and their use for risk prediction in the general population, as well as in high-risk groups. Following this, Sundström review other biomarkers from the cardiomyocyte, as well as the non-cardiomyocyte compartment of the myocardium. Pischon assesses the role of some of the most promising obesity biomarkers in cardiovascular epidemiology, such as fat-derived inflammatory markers, adiponectin, resistin, and fetuin-A. Halim and Newby turn our focus to the use of biomarkers for prognostication in patients with prevalent disease, and review the current state of knowledge in this field. Finally, Ikonomidis discusses the recent approach of combining biomarkers from different pathways for increased predictive and prognostic capability in so-called multimarker strategies. Taken together, we believe that this special issue gives a comprehensive overview of the contemporary area of circulating biomarkers in cardiovascular disease.

\section{References}

[1] Available at: http://www.merriam-webster.com/dictionary/ biomarker, Accessed 2009-05-25.

[2] W.B. Kannel, T.R. Dawber, A. Kagan, N. Revotskie and J. Stokes 3rd, Factors of risk in the development of coronary heart disease-six year follow-up experience, The Framingham Study, Ann Intern Med 55 (1961), 33-50.

[3] P.W. Wilson, R.B. D'Agostino, D. Levy, A.M. Belanger, H. Silbershatz and W.B. Kannel, Prediction of coronary heart disease using risk factor categories. Circulation 97(18) (1998), 1837-1847.

[4] R.M. Conroy, K. Pyorala, A.P. Fitzgerald, S. Sans, A. Menotti, G. De Backer, D. De Bacquer, P. Ducimetiere, P. Jousilahti, U. Keil, I. Njolstad, R.G. Oganov, T. Thomsen, H. TunstallPedoe, A. Tverdal, H. Wedel, P. Whincup, L. Wilhelmsen and I.M. Graham, Estimation of ten-year risk of fatal cardiovascular disease in Europe: the SCORE project, Eur Heart $J$ 24(11) (2003), 987-1003. 


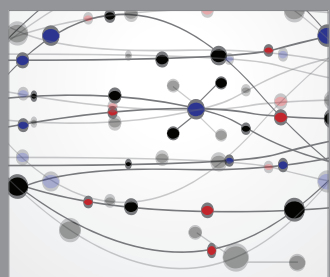

The Scientific World Journal
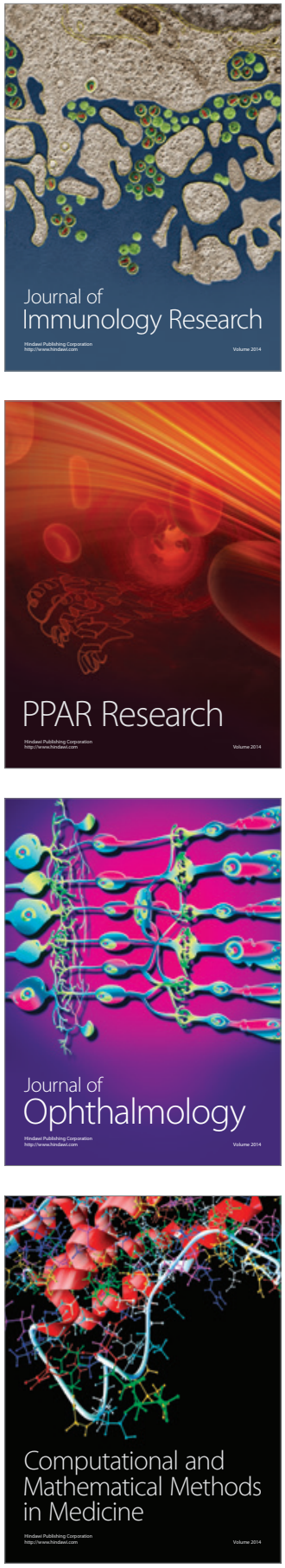

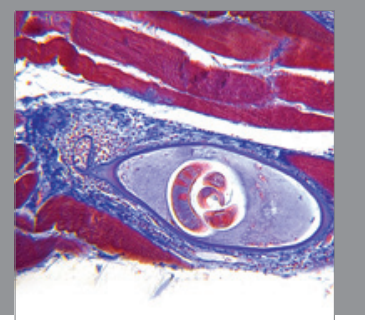

Gastroenterology

Research and Practice
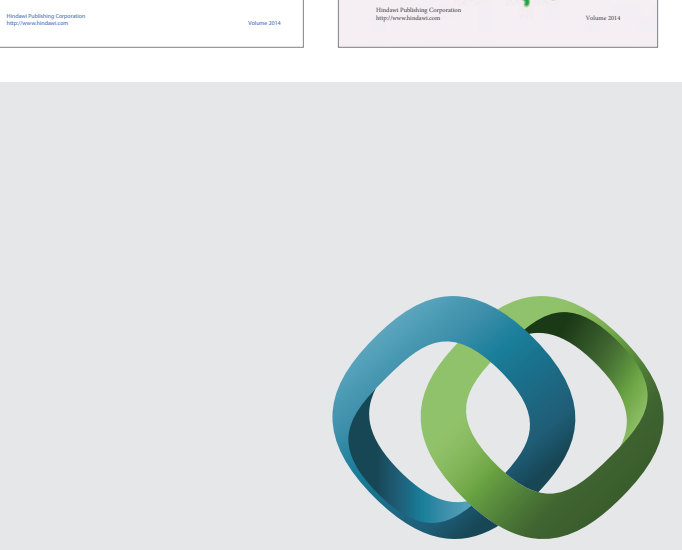

\section{Hindawi}

Submit your manuscripts at

http://www.hindawi.com
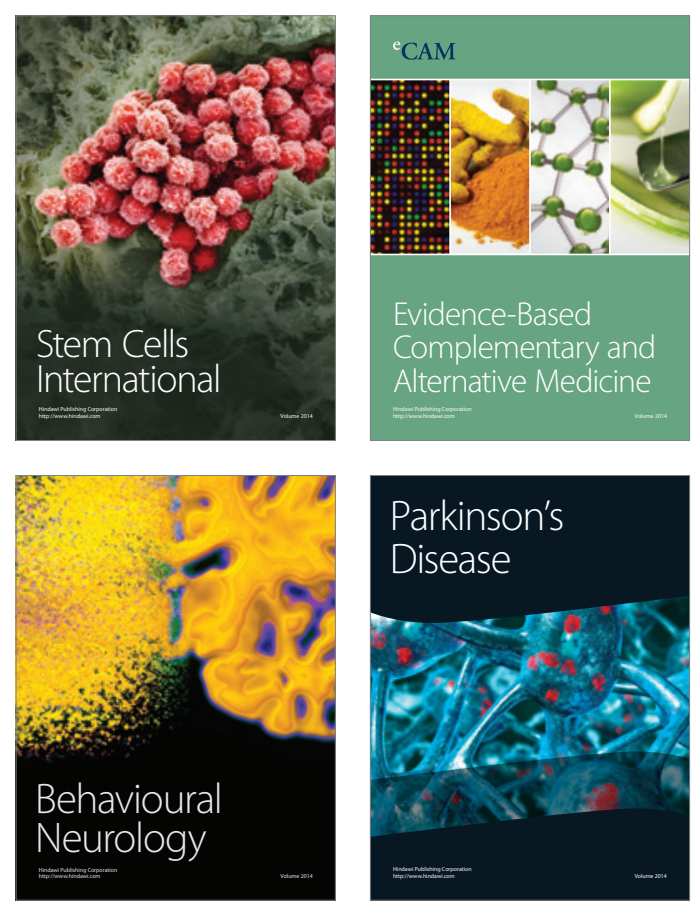

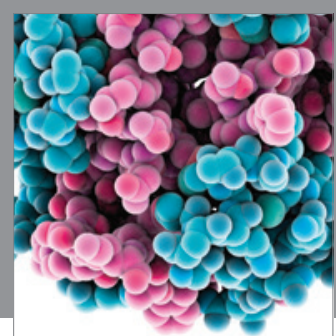

Journal of
Diabetes Research

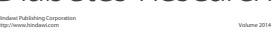

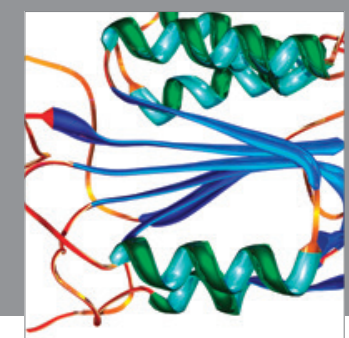

Disease Markers
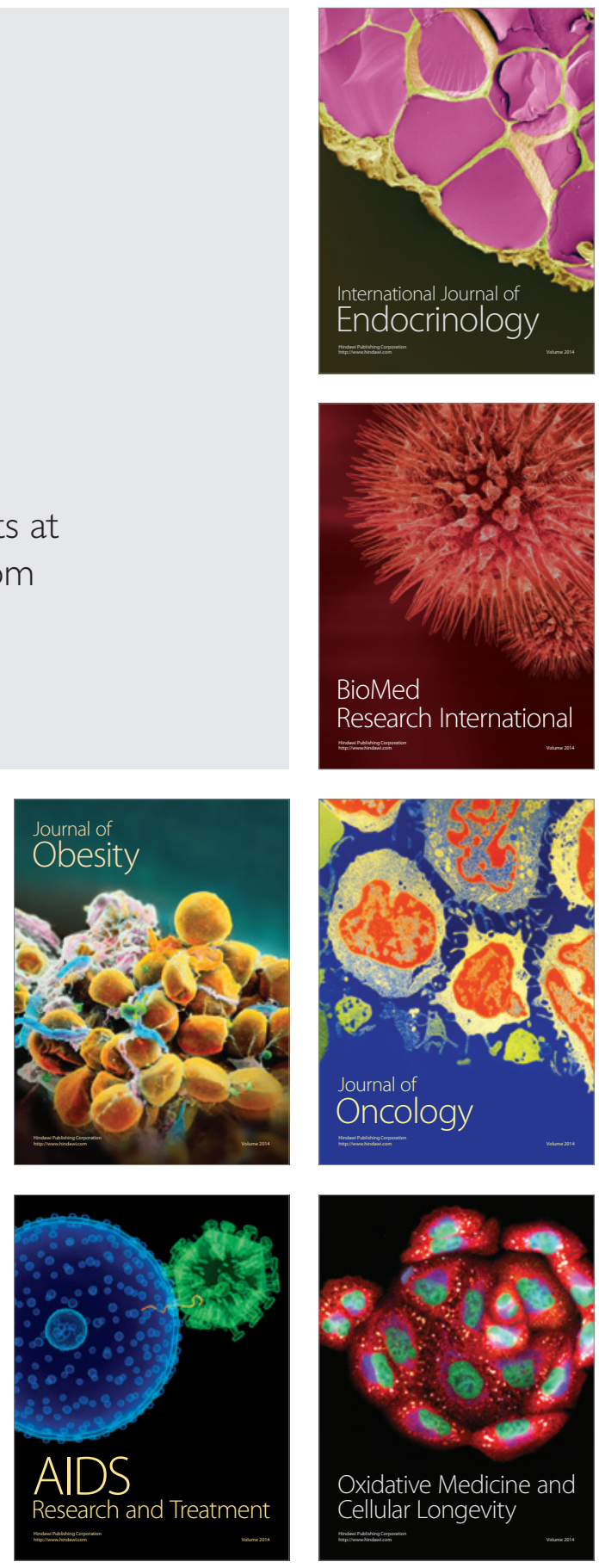